\title{
Droit et cultures
}

Revue internationale interdisciplinaire

73 | 2017-1:

De la famille aux familles

Dossier : De la famille aux familles

\section{L'adoption légale comme révélateur de l'évolution de la famille au Québec}

Legal Adoption as a Revealing Indicator of the Evolution of the Family in Quebec

\section{FranÇOISE-Romaine OUEllette et CARMEN LAVAllÉE}

p. $49-68$

\section{Résumés}

Français English

Cet article relie l'évolution de l'adoption au Québec aux transformations de la famille et du contexte social et normatif dans lequel elle s'inscrit. Il s'intéresse aux effets de l'adoption sur les liens familiaux antérieurs de l'enfant adopté. Il met plus particulièrement en lumière les particularités de l'adoption intrafamiliale, qui est méconnue. Trois périodes sont distinguées. La première (19241960) est caractérisée par l'exclusion des enfants naturels et adoptés de l'ordre familial légitime. La deuxième (1960-1980), par la transition vers un modèle de famille conjugale égalitaire qui a permis la légitimation des adoptés. La troisième (de 1980 à aujourd'hui) instaure un paradigme juridique strictement égalitaire, qui assure l'intégration familiale de tous les enfants au sein d'une famille idéalement formée de deux parents et qui exclut toute forme de pluriparenté. L'adoption devient substitutive en toutes circonstances.

This paper connects the evolution of adoption in Quebec to the broader social and normative context of family change. It underlines the effects of adoption on the adopted child's previous family ties. It more specifically highlights the peculiarities of family adoptions, which are largely unknown. Three periods are distinguished. The first (1924-1960) is characterized by the exclusion of illegitimate and adopted children from the legitimate family order. The second (1960-1980) period marks the transition to an egalitarian nuclear family model and the granting of similar rights to adopted and legitimate children. The third one (1980 until now) establishes a strictly egalitarian legal paradigm that provides all children with equal rights towards their parents and other relatives. It excludes any form of plural kinship. Adoption thus becomes substitutive at all time. 


\section{Entrées d'index}

Mots-clés : adoption, filiation, adoption intrafamiliale, adoption de l'enfant du conjoint, enfants illégitimes, enfants adoptés

Keywords : Adoption, Filiation, In-family adoption, Step-child adoption, Illegitimate children, Adopted children

\section{Texte intégral}

\section{Introduction}

L’adoption légale constitue un révélateur du degré de flexibilité qu'une société autorise, à un moment donné de son histoire, dans le tracé des affiliations familiales. Le présent article relie l'évolution de l'adoption au Québec à la transformation, au fil du temps, de la famille et du contexte social et normatif dans lequel elle s'inscrit. Il s'intéresse aux effets de l'adoption sur les liens familiaux antérieurs de l'enfant adopté, mettant en perspective la rupture complète qu'exige aujourd'hui l'adoption plénière. Plus particulièrement, il vise à mettre en relief l'adoption intrafamiliale, celle qui confie l'enfant à quelqu'un de sa parenté ou au conjoint de l'un de ses parents. Les adoptions de ce type n'étant répertoriées par aucune instance judiciaire ou administrative, elles sont méconnues. Les auteures s'appuient sur leur analyse ${ }^{1}$ d'un échantillon de dossiers judiciaires d'adoption du district de Saint-François ${ }^{2}$ entre 1953 et $2014^{3}$. Cette analyse a permis pour la première fois de repérer les adoptions intrafamiliales au sein d'un échantillon d'adoptions québécoises et de cerner leurs spécificités.

2 La première partie de l'article porte sur la période allant de 1924 à 1960, au cours de laquelle l'adoption concerne d'abord les enfants illégitimes. La loi ne leur confère que des droits limités, car elle porterait autrement atteinte à l'ordre familial légitime fondé sur le mariage et la puissance paternelle. La deuxième partie porte sur la transition des années 1960 et 1970, au cours desquelles un modèle de famille conjugale égalitaire se précise. En 1969, l'enfant adopté devient légitime et des obstacles législatifs à l'adoption intrafamiliale sont levés. Enfin, en 1982, l'adoption est intégrée au Code civil qui confère désormais les mêmes droits à tous les enfants dont la filiation est établie. La troisième partie de l'article examine donc comment l'instauration de ce paradigme juridique strictement égalitaire a transformé les usages de l'adoption.

\section{La préservation de l'ordre familial légitime (1924-1960)}

3 L'adoption légale a été permise dans la plupart des pays occidentaux après la Première guerre mondiale, dans une perspective d'aide à l'enfance. Au Québec, elle le sera à partir de 1924, dans une loi statutaire ensuite plusieurs fois modifiée, plutôt que par son insertion dans le Code civil. Du point de vue de l'assistance aux enfants, cette loi comportera longtemps des restrictions importantes à l'adoption, reflétant un contexte social et juridique qui condamnait toute transgression des frontières de la famille reconnue légitime.

$4 \quad$ Religion, parenté et famille légitime

5 La majorité franco-catholique du Québec est alors pauvre, peu scolarisée et n'a pas accès aux principaux leviers du pouvoir économique. Elle est fortement soumise à l'influence de l'Église, qui condamne la contraception et toute sexualité hors mariage. 
L’appareil étatique est peu développé. Ce sont des communautés religieuses catholiques, et des organisations privées protestantes et juives, qui administrent les établissements de santé et aident les indigents ${ }^{4}$. Jusqu'aux années 1960, même fragilisées par l'urbanisation accélérée 5 , les solidarités familiales assurent l'essentiel de la protection sociale. Les orphelins sont placés de préférence dans leur parenté et l'adoption de fait de l'enfant privé de parents est relativement commune. Par contre, l'enfant est un don de Dieu que sa mère ne peut redonner à un tiers, à moins d'y être contrainte ${ }^{6}$.

7 Ce modèle familial coexiste avec un droit de la famille organisé autour du mariage et de la puissance paternelle. Bien qu'inspiré du Code Napoléon, il a retenu du droit anglais la liberté illimitée de tester7. De plus, il est resté fidèle à l'ancien droit français en n’imposant qu'un minimum de responsabilité aux parents naturels ${ }^{8}$. Le mariage entraîne pour les époux l'obligation de nourrir, élever et entretenir les enfants nés de leur union. Ces derniers ont le statut d'enfants légitimes et sont soumis à la puissance paternelle. Ils sont inscrits de plein droit dans la parenté de leurs auteurs et jouissent de droits alimentaires et successoraux plus étendus que ce n'est le cas aujourd'hui : l'enfant légitime est non seulement l'héritier légal et le créancier ou débiteur alimentaire de ses parents et de ses autres ascendants, mais aussi de ses beaux-parents9.

8 L'enfant né en dehors des liens du mariage est illégitime. À moins de légitimation par mariage ${ }^{10}$, il n'aura droit qu'à des aliments de la part de ses parents naturels, s'ils sont connus. Il pourra recevoir d'eux une donation entre vifs ${ }^{11}$ ou par testament, mais il n'est pas leur héritier légal, car il n'appartient pas juridiquement à leur parenté. Le Code civil du Bas-Canada consacre ainsi une vision patrimoniale de la famille légitime et ne reconnaît pas l'existence de la famille naturelle ${ }^{12}$.

\section{La prise en charge des enfants illégitimes}

9 Dans un tel contexte culturel et juridique, qui exclut l'enfant illégitime de l'ordre familial, l'enjeu est de déterminer qui va en assumer la charge. Il arrive qu'il soit gardé dans sa famille naturelle ou adopté de fait ou même qu'il soit reconnu comme l'enfant d'une tante ou d'une grand-mère, par exemple. Toutefois, dans la grande majorité des cas $^{13}$, il est abandonné dans l'une des crèches tenues par les communautés religieuses. L'État n'offre aucun soutien aux mères célibataires ${ }^{14}$ et le système de justice rend très difficiles les poursuites en reconnaissance de paternité ${ }^{15}$. Le droit canonique ignore l'adoption, mais l'appartenance par le baptême à la parenté " des enfants de Dieu » ${ }^{16}$ justifie d'abandonner «l'enfant du péché », à condition qu'il soit élevé dans la foi catholique, afin d'éviter le déshonneur et de réhabiliter la " fille-mère ${ }^{17}$. Idéalement, il sera recueilli par des époux charitables.

10 Leurs crèches étant surpeuplées, les communautés religieuses ont été d'ardents promoteurs de l'adoption légale afin de faciliter le recrutement de parents d'adoption. Toutefois, les autorités ecclésiastiques se sont opposées à la Loi concernant l'adoption votée en $1924^{18}$, notamment parce qu'elle autorisait implicitement l'adoption d'un enfant légitime. Elle transgressait à leurs yeux l'ordre naturel et divin en vertu duquel la puissance paternelle était concédée au père. De plus, elle ne protégeait pas la foi religieuse de l'enfant et ne faisait pas intervenir l'institution religieuse à qui il avait été confié. Les parlementaires ont cédé et modifié leur loi dès $1925^{19}$.

\section{Une adoption réservée à l'enfant illégitime ou abandonné}

11 La loi remaniée de 1925 impose l'identité de foi religieuse et un écart d'au moins vingt ans d'âge entre l'adoptant et l'adopté (art. 4), qui doit être mineur (art. 6). Elle permet l'adoption aux seuls époux faisant vie commune et à la personne veuve ou célibataire sans enfant qui adopte un enfant du même sexe qu'elle (art. 3). 
12 Le droit de consentir à l'adoption est désormais réservé au père (à défaut, à la mère) ou au tuteur de l'enfant illégitime. Les seuls enfants légitimes qui peuvent être adoptés n'ont aucun parent en mesure de consentir : les orphelins de père et de mère ou dont les père et mère, ou le survivant, sont irrémédiablement privés de raison, si aucun autre ascendant ne peut en prendre soin (art. 1 d, par. 3). L'enfant illégitime abandonné de fait ou dans une institution de charité, pendant au moins deux ans, peut aussi être adopté sans consentement parental (art. 9). Si ses parents sont inconnus, le délai ne s'applique pas et le consentement est donné par l'institution de charité qui l'a recueilli (art. 8 d). En 1927, ce délai sera réduit à six mois et il deviendra aussi possible d'adopter sans consentement parental l'enfant légitime laissé en institution en qualité d'indigent depuis plus de deux ans (art. 13). Par ailleurs, en 1933, il sera aussi permis à un veuf ou une veuve ayant des enfants d'adopter un enfant de sexe différent, en cas d'adoption de fait antérieure au décès de son conjoint (art. 3b).

\section{La création d'un lien parent-enfant, sans véritable légitimation}

Après l'adoption, les parents sont privés de tous leurs droits et dispensés de toutes leurs obligations légales à l'égard de l'enfant (art. 16 par. 1). Celui-ci est considéré « comme l'enfant propre de ses parents d'adoption » (art. 16 par. 2) et le juge peut ordonner qu'il prenne le nom de l'adoptant (art. 17). Dès 1933, la transcription du jugement au double registre des actes de baptême, de mariage et de décès ne comporte aucune mention de l'illégitimité ou de l'adoption et ne mentionne que les parents adoptifs (art. 26).

Ces effets juridiques relèvent du modèle substitutif de l'adoption plénière. L'affirmation doit pourtant être nuancée, car cette adoption est révocable (art.19) et ne crée qu'un lien personnel entre l'adoptant et l'adopté, dont les effets ne s'appliquent à personne d'autre ${ }^{20}$. L'adopté n'entre pas dans la parenté de ses père et mère adoptifs, même s'il est soumis à la puissance paternelle. Il leur succède s'ils meurent $a b$ intestat, mais pas à leurs ascendants ou alliés. Par contre, ces derniers héritent des biens que l'adopté a pu recevoir d'eux, ainsi que de ceux qu'il a acquis par lui-même (art.18 a). Il est soumis à des empêchements de mariage dans sa parenté naturelle ${ }^{21}$, mais à aucun dans la famille des adoptants : la fille adoptée peut épouser son frère adoptif ou son père adoptif devenu veuf, par exemple.

À cette époque, il est conseillé de déclarer l'enfant naturel comme étant né de parents inconnus pour faciliter son adoption ${ }^{22}$, puisque le certificat de baptême initial demeure accessible et peut encore être délivré par mégarde. De plus, les parents adoptifs préfèrent occulter l'absence de liens de sang entre eux et leur enfant en taisant l'adoption. Même s’ils conseillent de dire la vérité à l'enfant pour qu'il ne l'apprenne pas par d'autres, les travailleurs sociaux eux-mêmes appliquent un modèle d'adoption fermée, c'est-à-dire confidentielle et sans aucun contact entre les parents d'origine et d'adoption. Or, l'adoption laisse subsister des droits à l'égard de la famille naturelle, dont l'exercice est ainsi compromis. Au décès de l'adopté, les biens qu'il a acquis de ses parents et alliés naturels doivent être déférés de la même manière que s'il n'avait pas été adopté (art. 18, 2 b). Le droit n'ignore donc pas tout à fait sa parenté naturelle ${ }^{23}$, tout en le maintenant à la marge de sa parenté adoptive.

\section{L'adoption par le système des crèches}

Dès la fin des années 1930, la grande majorité des enfants adoptés l'ont été en préservant l'anonymat de la mère naturelle, à travers le système des crèches. Ce système s'est généralisé dans les années 1940 avec la création des agences confessionnelles d'adoption et la professionnalisation du service social, puis avec l'obligation faite aux hôpitaux de déclarer toutes les naissances illégitimes en 1949 et la création, en 1950, des agences diocésaines de services sociaux ${ }^{24}$. Dans les années 1950, il y avait au Québec seize 
crèches. Sur les 2700 enfants recueillis par année, environ 2500 étaient adoptés, les autres étant transférés dans un orphelinat vers l'âge de six ans ${ }^{25}$. La prédominance de ce type d'adoption s'illustre dans les dossiers étudiés dans le district judiciaire de SaintFrançois. En effet, 98,4 \% des 258 dossiers d'adoption enregistrés dans ce district, en 1953, concernent des enfants adoptés par des étrangers à la famille.

\section{L'adoption au sein du cercle familial}

17 Une facette peu connue de l'adoption à cette époque est qu'elle pouvait être le fait des père et mère naturels. En effet, les personnes appelées à consentir à l'adoption pouvaient elles-mêmes se porter requérantes. La mère pouvait adopter, avec son époux, son enfant naturel conçu avec un autre homme ou adopter seule sa fille naturelle. Un père pouvait adopter son enfant naturel avec son épouse ou adopter seul son fils nature ${ }^{26}$. Le père et la mère une fois mariés ensemble pouvaient adopter leur enfant naturel. L'enfant, même adultérin $^{27}$, sortait ainsi de l'illégitimité, bien que son adoption ne créait aucun lien avec les ascendants et collatéraux de ses père et mère.

18 L'adoption par un membre de la parenté pouvait notamment être le fait des grandsparents. Quand, en 1939, le consentement à l'adoption par un parent légitime est enfin permis, c'est pour le veuf ou la veuve qui consent en faveur des père et mère de son conjoint décédé. L'enfant occupera donc la position généalogique laissée vacante par le parent légitime dont il est orphelin. Suivant une logique patrimoniale, il sera un héritier de remplacement pour ses grands-parents. Il pourra en hériter à la fois à titre de fils (ou de fille) adoptif et à titre de petit-fils (ou petite-fille) légitime ${ }^{28}$.

En 1953, ces adoptions intrafamiliales sont rares, dans le district judiciaire de SaintFrançois. Seulement quatre enfants en font l'objet. L'un est adopté par ses parents maintenant mariés, deux autres par la mère naturelle et son époux, et le quatrième par un couple qui pourrait être les grands-parents maternels (ils ont six enfants et la mère naturelle porte leur nom). Ce très petit nombre de cas montre que si l'on transgresse souvent l'interdiction religieuse d'avoir des relations sexuelles hors mariage, l'obligation d'abandonner à la crèche l'enfant illégitime est encore très largement respectée dans les années 1950.

Dans les décennies qui vont suivre, l'ambiance cléricale et conservatrice des années 1940-1960 va se dissiper brusquement. L'adoption ne sera plus appelée à cacher l'opprobre d'une naissance illégitime, mais à s'articuler à un nouvelle perspective juridique sur la conjugalité et sur la responsabilité sociale à l'égard des enfants, qui donnera une place jusque là inédite à l'adoption intrafamiliale.

\section{La démocratisation des liens familiaux, 1960-1980}

$\mathrm{Au}$ cours des années 1960, les valeurs et les styles de vie se diversifient. La société québécoise vit une transformation profonde de ses institutions. Cette transition se préparait depuis au moins l'entre-deux-guerres ${ }^{29}$. Néanmoins, le contraste avec la dynamique sociale des années antérieures est si grand qu'on parlera de " révolution tranquille ».

On assiste au développement accéléré d'un État-providence ${ }^{30}$ qui déploie des services universels, sécularisés et gratuits d'éducation, de santé et de protection sociale. Des mesures publiques de redistribution garantissent plus d'autonomie aux individus, favorisant une nucléarisation de la famille. L'Église perd son influence. Des professionnels de la santé et des sciences humaines prennent le relais des communautés religieuses, qui cèdent à l'État leurs établissements. Divers mouvements de libération prennent leur essor, 
notamment le féminisme qui aura une influence majeure sur le droit de la famille québécois.

\section{De la parenté légitime à la famille conjugale}

Les prescriptions de l'Église en matière de mariage et de contraception sont délaissées au profit d'une nouvelle idéologie de l'amour conjugal valorisant l'intimité affective et l'épanouissement sexuel. Le modèle du couple ménagère/pourvoyeur reste dominant, mais plusieurs femmes conservent une activité professionnelle après le mariage. Les indices de fécondité subissent une baisse spectaculaire de $47 \%^{31}$. Entre 1960 et 1970, le nombre moyen d'enfants par famille passe de 4 à $2^{32}$.

En 1964, le législateur abroge la puissance maritale et stipule que la femme concourt avec son mari à la direction morale et matérielle de la famille (art. 174 C.c.B.C.). Les mères célibataires obtiennent l'accès à l'aide sociale, ce qui leur permet d'élever seules leur enfant naturel. En 1968, une loi fédérale rend le divorce applicable partout au Canada et donc, aussi au Québec33. La même année, le Québec permet la célébration d'un mariage civil, de sorte que les catholiques divorcés pourront se remarier 34 .

Les crèches et la plupart des orphelinats ferment 35 , non sans avoir dû confier en adoption internationale annuellement de trois à quatre cents enfants (en France et aux États-Unis, principalement) entre 1965 et $1970^{36}$. La famille est désormais reconnue comme le meilleur milieu de vie pour l'enfant orphelin ou abandonné. Il n'est plus socialement acceptable de l'en exclure. Elle est aussi revendiquée comme champ d'expertise par les professionnels des services sociaux. Or, au cours des années 1970, la famille est perçue comme étant en crise.

La monoparentalité et les unions libres sont en hausse. Les taux de divortialité qui étaient de 8\% en 1968 augmentent à 38\% en 1976. Les femmes envisagent la maternité comme une expérience qu'il est possible de refuser et la fécondité subit une chute marquée ${ }^{37}$. Le recours à la stérilisation contraceptive, surtout féminine, monte en flèche dès $1971^{38}$.

Dans ce contexte, une perspective moins normative et plus sociologique sur la famille se généralise, s'intéressant essentiellement au ménage nucléaire sans faire référence à l'univers plus large d'une parenté solidaire39. Elle se veut inclusive des ménages monoparentaux, des couples concubins, des parents divorcés et des familles adoptives. Le principal problème social désormais associé à la famille est celui de l'impact potentiellement négatif pour les enfants des unions de fait, de l’instabilité des couples et de l'incertitude quant à la place du père après la rupture. La prise en charge de l'enfant se précise d'ailleurs comme étant l'enjeu en fonction duquel l'État et les divers spécialistes de l'enfant et de son développement peuvent exercer une surveillance et un contrôle sur les parents et les familles ${ }^{40}$.

\section{Vers une normalisation de l'adoption et de la famille adoptive}

Reflétant ces transformations, l'adoption évolue vers un élargissement des circonstances où elle peut être autorisée et une intégration complète de l'enfant adopté dans sa famille. En 1960, le modèle de l'adoption fermée est renforcé, tout en éliminant certains freins à l'adoption intrafamiliale ${ }^{41}$. Les dossiers judiciaires d'adoption deviennent confidentiels (art. 29). L'adoption des majeurs adoptés de fait est permise, ainsi que celle de l'enfant adultérin abandonné (art. 3 par. 5 et 6). Le veuvage d'un grand-parent ne fait plus obstacle à l'adoption d'un petit-enfant naturel de sexe différent (art. 11). De plus, l'obligation d'une différence de vingt ans d'âge entre l'adoptant et l'adopté est abolie en cas d'adoption de 
son propre enfant (art. 4). La loi commence ainsi à tenir compte du fait que les gens qui adoptent veulent former une famille semblable aux autres.

Les adoptions du district de Saint-François, en 1963, reflètent ces changements. Cette année-là, les adoptions ont beaucoup diminué par rapport à 1953 (145 contre 258). Par contre, $12,4 \%$ sont maintenant intrafamiliales. Presque la moitié de celles-ci sont des adoptions par la parenté (8) et les autres (10) des adoptions de leur propre enfant par l'un ou l'autre des parents ou par les deux. Grâce aux amendements de 1960, trois adoptions de fait de majeurs $(22,24$ et 27 ans) sont légalisées et une veuve adopte son petit-fils.

En 1964, le parent survivant de l'enfant légitime orphelin de père ou de mère peut consentir à son adoption par son nouveau conjoint et reformer ainsi une famille biparentale (art. 6 par. 4).

\section{L'adoption, une responsabilité d'ordre public}

31 Une refonte de la loi en $1969^{42}$ donne préséance à l'intérêt de l'enfant et confie la responsabilité des adoptions à des sociétés sans but lucratif mandatées par le ministre de la Famille et du bien-être social. Le placement d'un enfant sans passer par leur intermédiaire est cependant permis, à condition que le ministre en soit avisé, ce qui facilitera les adoptions intrafamiliales et les premières adoptions internationales.

Cette loi autorise l'adoption de tout enfant dont le parent consent à l'adoption, ou dont aucun ascendant ne s'occupe de fait, y compris de l'enfant légitime dont le père naturel n'est pas le mari de la mère (art. 7f)43. Le tribunal peut passer outre à l'identité de foi religieuse en cas d'adoption de fait (art. 5) ou autoriser qu'une personne seule adopte son propre enfant ou petit-enfant de sexe différent (art. $3 \mathrm{~d}$ ). Les époux séparés de corps, ou l'un d'eux, peuvent adopter l'enfant qu'ils ont adopté de fait avant leur séparation (art. 3 c). L'enfant légitime de parents divorcés ou dont le mariage a été dissout peut être adopté par le nouveau conjoint de son père ou de sa mère avec le consentement de l'autre parent (art. 7c). L'époux ou l'épouse du requérant doit se porter partie à la requête et adopte donc son propre enfant légitime (art. 20).

\section{La légitimation de l'enfant adopté}

L'enfant adopté devient légitime à tous égards et à l'égard de tous (art. 38) et son adoption n'est plus révocable. Il jouit des mêmes droits que l'enfant né dans le mariage. Les procédures doivent éviter tout contact entre parents d'origine et d'adoption et ce sont autant les dossiers administratifs que judiciaires d'adoption qui sont dorénavant confidentiels (art. 30 et 31).

Rien dans la loi de 1969 ne précise une rupture des liens antérieurs qui serait plus complète qu'auparavant ${ }^{44}$. En revanche, l'enfant adopté né de parents mariés ne pourra plus prouver sa légitimité antérieure, car l'acte de naissance initial n'est plus accessible. Même adopté par ses grands-parents, il aura perdu sa qualité de fils ou de fille de ses parents antérieurs. L'article 39 de la loi prévoit par contre une exception : le nouveau certificat de naissance sera rédigé suivant une formule distincte qui mentionne les parents d'origine et d'adoption dans le cas de l'enfant légitime adopté par le nouveau conjoint de l'un de ses parents. Il pourra faire ainsi valoir ses droits successoraux dans sa famille légitime d'origine 45 . L'adoption de l'enfant du conjoint est donc clairement identifiée comme additive ; elle crée une forme de pluriparenté.

\section{Émergence de l'adoption de l'enfant du conjoint comme pratique distincte}


Dans le district de Saint-François, les changements sociaux et législatifs des années 1960 ont provoqué une hausse importante des adoptions intrafamiliales qui ont plus que triplé. En effet, le nombre total d'adoptions réalisées, en 1973, est le même (145) que dix ans plus tôt, mais 41,6\% (60) d'entre elles sont maintenant des adoptions intrafamiliales. Elles touchent, entre autres, dix-huit enfants légitimes qui seront ainsi dotés d'une double filiation. Treize d'entre eux sont adoptés par le nouveau conjoint de leur mère maintenant divorcée. Cinq autres (une fratrie de 3 enfants et une autre de 2 enfants) sont adoptés par leurs propres parents, qui les ont élevés, alors que légalement leur père était l'ex-époux de leur mère. Les autres sont comme auparavant des enfants naturels adoptés par leurs parents, par leur seule mère ou par celle-ci et son époux afin de les légitimer. Les quatre enfants adoptés dans leur parenté, dont un homme de 37 ans par sa sœur qui l'a élevé, sont des enfants naturels.

\section{D'institution de filiation à mesure de protection de l'enfant}

À la fin des années 1970, la Loi sur la protection de la jeunesse46 (ci-après LPJ), en vigueur en 1979, reconnaît l'enfant comme sujet de droit à part entière. Elle précise les motifs qui peuvent justifier une ingérence de l'État, qui ne devra plus se limiter à un rôle de surveillance et de contrôle, dans le champ des responsabilités parentales. La loi désigne le Directeur de la protection de la jeunesse (DPJ) comme seul responsable des placements d'enfants en vue d'adoption, abolissant la possibilité d'un placement privé, sauf quand l'adoptant est un proche parent ou le conjoint du parent, ou quand il transige avec un organisme reconnu d'adoption internationale. Le DPJ peut aussi demander au tribunal de déclarer admissible à l'adoption l'enfant dont les parents n'ont pas assumé le soin, l'entretien et l'éducation pendant au moins six mois (art. 71 LPJ). L'adoption est ainsi définie comme une mesure de protection de l'enfant et, dans ce but, des parents jugés inadéquats peuvent être disqualifiés en tant que parents.

La même année, le principe de la puissance paternelle est remplacé par celui de l'autorité parentale (art. 243 C.c.B.C. devenu art. 600 C.c.Q.). L'autorité parentale détermine moins des droits sur l'enfant que des devoirs et des obligations 47 à son égard, dans son meilleur intérêt. Les parents l'exercent conjointement, peu importe leur statut matrimonial. Cette nouvelle disposition consacre l'égalité des père et mère, mais concentre aussi sur eux seuls la responsabilité de l'enfant. Le législateur définit ainsi une famille juridique biparentale constituée par la présence d'un enfant à charge. Ce modèle biparental, construit à partir d'une notion de responsabilité parentale exclusive, exclut l'éventuel beau-parent. Cependant, la loi prévoit la déchéance du titulaire de l'autorité parentale en cas de comportement répréhensible ou d'abandon volontaire, ce qui l'empêchera de s'opposer à ce que l'autre parent consente à l'adoption de leur enfant en faveur de son nouveau conjoint (art. 606 C.c.Q.)

De 1960 à 1980, le processus de démocratisation des liens familiaux aura ainsi déconstruit le modèle antérieur de la parenté légitime, accordant égalité et autonomie aux parents, tout en plaçant l'enfant à l'interface entre la sphère familiale privée et l'autorité publique protectrice de l'enfant. L'évolution de l'adoption allait forcément être conditionnée par cette position d'interface. Un point de bascule dans la reconnaissance de droits égaux à l'enfant, y compris à l'enfant adopté, sera atteint avec la réforme du droit de la famille qui sera mise en place au début des années 1980. À partir de là, de nouveaux usages de l'adoption vont se développer. 


\section{Un droit familial égalitaire (de 1980 à aujourd'hui)}

En 1980, après une tournée provinciale de consultation, l'Assemblée nationale du Québec vote la Loi instituant le Code civil et portant réforme du droit de la famille ${ }^{48}$. Le principe juridique d'égalité garanti depuis 1975 par la Charte des droits et libertés de la personne 49 est au cœur de cette réforme qui consacre l'égalité des sexes et l'égalité des filiations.

Tous les enfants dont la filiation est établie ont désormais les mêmes droits, peu importe les circonstances de leur naissance (art. 522 C.c.Q). Les notions de légitimité et d'illégitimité ont donc disparu des textes. La famille implicitement reconnue par le droit prend ainsi une extension nouvelle. Puisque la condition de mariage des parents n'existe plus, il n'y a plus de différence discriminante entre parents mariés, en union de fait, célibataires ou divorcés, ni entre familles biparentales, monoparentales ou recomposées, ce qui se reflétera rapidement dans le droit social et fiscal. Par contre, la parenté susceptible d'être tenue responsable de verser des aliments à l'enfant se voit réduite : l'obligation alimentaire réciproque est maintenant limitée aux ascendants en ligne directe $^{50}$.

La Loi sur l'adoption est abrogée et les dispositions qui définissent les conditions et les effets de l'adoption sont désormais intégrées au Code civil. L'adoption devient, avec la filiation par le sang, l'un des deux modes d'établissement de la filiation. La filiation est maintenant ancrée, tantôt dans sa dimension volontaire, tantôt dans sa dimension biologique. Elle est cependant toujours exclusive : chaque enfant ne peut avoir qu'un seul père et qu'une seule mère. Ce principe d'exclusivité que véhiculaient implicitement le système des crèches, l'adoption fermée et le secret entretenu par les familles, est rendu explicite et contraignant dans les dispositions portant sur la filiation adoptive, qui ne permettent plus de nuancer les effets de l'adoption en fonction des circonstances qui la justifient.

\section{Une adoption substitutive pour tous ${ }^{51}$}

Toute personne majeure peut adopter, seule ou conjointement (art. 546 C.c.Q.), mais il n'est plus permis d'adopter son propre enfant. Le consentement parental à l'adoption peut être général ou spécial. Le consentement général entraîne la remise de l'enfant au DPJ, qui choisit alors les adoptants. Le consentement spécial est donné en faveur d'un ascendant, d'un parent en ligne collatérale jusqu'au 3e degré, ou d'un conjoint de cet ascendant ou parent (art. 555 C.c.Q.). L'enfant dont les parents n'ont pas assumé les soins, l'entretien et l'éducation depuis au moins six mois peut faire l'objet d'une déclaration judiciaire d'admissibilité à l'adoption (art. 559 C.c.Q.). Le fait qu'un autre ascendant que le père ou la mère puisse s'en occuper ne s'oppose plus à qu'il soit déclaré adoptable. Au contraire, l'admissibilité à l'adoption peut être demandée par un ascendant, un parent en ligne collatérale jusqu'au 3e degré, un conjoint de cet ascendant ou parent, par l'enfant luimême, s'il est âgé de quatorze ans, ou par le DPJ (art. 560 C.c.Q.). Comme pour le consentement spécial, le conjoint ou la proche parenté peut donc encore intervenir dans l'adoption de manière autonome, sans l'intermédiaire du DPJ.

43 L'adoption confère à l'adopté les mêmes droits que la filiation par le sang, mais elle devient substitutive, pour tous : les effets de la filiation antérieure prennent fin et l'adopté n'appartient plus à sa famille d'origine (art. 577 C.c.Q.). C'est à travers cette substitution que l'autorité parentale, qui découle de la filiation, est transférée aux adoptants. Cependant, lorsqu'un parent consent à l'adoption en faveur de son conjoint, le lien de filiation entre ce parent et son enfant est maintenu. Seul le conjoint adoptant est requérant 
à l'adoption et seul le lien de filiation avec le parent qu'il remplace, s’il était légalement reconnu, se trouve rompu (art. 579 C.c.Q.).

L'adopté est doté d'un nouvel état civil et son acte de naissance ne fait mention ni de l'adoption ni des parents d'origine. Au nom de l'égalité de tous les enfants, malgré les recommandations formulées à l'époque par l'Office de révision du Code civil, rien n'est conservé des dispositions de 1969 qui laissaient subsister, sur le certificat de naissance et au niveau des droits successoraux, la filiation d'origine de l'enfant adopté par le conjoint de son père ou de sa mère.

Les demandes d'accès au contenu des dossiers d'adoption s'étant multipliées et ayant été autorisées par certains juges, une nouvelle disposition permet à l'adopté de 18 ans et plus $^{52}$ ou représenté par ses parents adoptifs d'obtenir sur demande un sommaire des renseignements non identifiants figurant à son dossier. Il peut aussi retrouver ses parents d'origine s'ils ont préalablement consenti à ce que leur identité lui soit révélée (art. 583 C.c.Q.). Ces derniers peuvent aussi connaître les antécédents de leur enfant biologique âgé de 18 ans ou plus s'il y consent. Ce droit de rechercher ses origines constitue encore maintenant le seul contrepoids prévu par le Code civil à la rupture de la filiation d'origine. Or, de plus en plus d'enfants adoptés arrivent à l'adoption après un parcours de vie familiale qui justifierait de ne pas occulter leur identité et leurs liens antérieurs.

\section{L'adoption, forme exemplaire de lien électif}

Depuis 1980, les candidats à l'adoption ont toujours été plus nombreux que les enfants disponibles pour l'adoption. À la fin de la décennie, ils sont environ 1500 en attente d'être évalués par le DPJ et s'insurgent contre les délais qu'ils subissent. Leurs revendications et leurs efforts pour promouvoir l'expérience de la parentalité adoptive seront d'importants révélateurs de la prégnance du modèle de parenté élective qui s'est mis en place à cette période ${ }^{53}$. Ce modèle met en valeur le libre choix d'une personne ou d'un couple de s'engager dans un projet parental, ainsi que la réalisation de soi à travers la relation affective parent-enfant. Il valorise le désir d'enfant dégagé du désir d'être uni par le sang, potentiellement ouvert à l'adoption d'enfants de profils variés ou à l'accueil des gamètes d'un tiers en cas de procréation assistée.

47 L'adoption internationale, qui augmente en flèche de 1990 à 2004 avant de décliner progressivement 54 , sera le contexte privilégié de mise en valeur de ce modèle électif. Elle touchera des enfants de tous âges et origines ethnoculturelles. Les adoptions internes sous le contrôle du DPJ feront appel à ce même modèle, dans le but d'assurer la protection de l'enfant, en exigeant des candidats qu'ils s'engagent d'abord comme famille d'accueil rémunérée d'un enfant qui a été retiré de son milieu familial, qui peut être déjà grand, avoir une fratrie, avoir bien connu ses parents. Ils pourront l'adopter par la suite si ce dernier s'attache à eux et qu'ils deviennent ses parents psychologiques. Ses parents d'origine devront être jugés incapables de le reprendre en charge pour que le tribunal le déclare admissible à l'adoption55. Depuis 2008, dans le district de Saint-François, le nombre des adoptions sous le contrôle du DPJ oscille entre 24 et 38 cas annuellement et il est toujours deux, trois ou quatre fois plus élevé que celui des adoptions internationales (entre 5 et 12 annuellement).

48 Le même idéal de parentalité élective colore d'autres formes d'adoption, mais elles répondent aussi, ou d'abord, à d'autres motivations.

\section{Les adoptions intrafamiliales}

49 Les adoptions intrafamiliales s'inscrivent dans la dynamique particulière d'une famille étendue ou dans celle d'une recomposition familiale. Après 1980, n'étant pas répertoriées, elles sont demeurées tout aussi méconnues qu'auparavant. Elles sont donc largement 
oubliées lors des réflexions sur l'adoption parce qu'on croit qu'elles sont marginales. Or, il est permis, au contraire, d'avancer que leur part dans l'adoption au Québec est relativement importante.

En effet, dans le district de Saint-François, les adoptions intrafamiliales représentent au moins le tiers de toutes les adoptions internes dans 8 des 10 années postérieures à 1980 que nous avons étudiées. Sauf en 1993 où elles se chiffrent à $63 \%$ des adoptions internes, elles sont toujours plus nombreuses (ou égales, en 2009) que les adoptions internationales.

51 Les adoptions par un membre de la parenté (entre 2 et 8 annuellement) sont majoritairement le fait des grands-parents maternels ${ }^{56}$. Elles sont motivées par la fragilité ou l'inadéquation des père et mère, même si ces derniers conservent parfois la garde de leurs autres enfants. Elles découlent d'un consentement à l'adoption ou, moins souvent, d'une déclaration d'admissibilité à l'adoption, mais rarement du décès d'un parent et très rarement de sa déchéance. Elles sont parfois réalisées sous le contrôle du DPJ, ce qui indique une certaine réduction de la zone d'autonomie réservée à la parenté dans l'adoption.

52 Les adoptions de l'enfant du conjoint (entre 7 et 20 annuellement) sont les plus fréquentes. Dans moins de la moitié des cas répertoriés après 1980 (sur 10 années), elles donnent un père à l'enfant qui n'en n'a jamais eu. Elles poursuivent un peu plus souvent une logique de substitution du parent non-gardien par le beau-parent dans une famille recomposée ${ }^{57}$. En droit québécois, il n'existe pas d'autre alternative pour faire reconnaître des droits à ce beau-parent à l'égard de l'enfant qu'il élève comme le sien. Le parent remplacé est décédé ou déchu, mais a rarement consenti à l'adoption. Malgré les graves conséquences de cette rupture des liens, la loi ne prévoit ni l’intervention du DPJ, ni une évaluation psychosociale pour vérifier qu'elle est dans l'intérêt de l'enfant ${ }^{8}$. Depuis 1994, le consentement spécial peut être donné en faveur d'un conjoint de fait à condition d'une durée de cohabitation d'au moins trois ans (art 555 C.c.Q.). Le Code civil ne désigne pas encore expressément les conjoints de même sexe, mais le droit de ces derniers d'inscrire un enfant dans une filiation homoparentale leur sera reconnu quelques années plus tard.

\section{L'adoption homoparentale}

Jusqu'en 2002, les conjoints homosexuels n'ont pu adopter qu'un enfant confié par le DPJ ou un enfant d'un pays étranger, à titre de célibataires, en cachant leur vie de couple et en se résignant à ce qu'un seul d'entre eux ait un lien légal avec l'enfant. En 2002, la Loi instituant l'union civile et établissant de nouvelles règles de filiation ${ }^{59}$ permet que des personnes de même sexe soient désignées comme les parents d'un enfant sur son acte de naissance (art. 115 C.c.Q).

La coupure avec le modèle de la famille légitime est ainsi tout à fait complétée. Le changement confirme et consolide l'égalité des conjoints dans un couple et l'égalité des filiations. L'adoption ne sera plus dès lors refusée aux couples gais et lesbiens, qu'ils soient candidats à l'adoption d'un enfant placé ou que l'un des conjoints consente à ce que l'autre adopte son enfant. Plusieurs contourneront ainsi l'interdiction qui leur est imposée encore aujourd'hui par la grande majorité des pays d'origine des enfants. L'un adopte seul l'enfant à l'étranger et consent, par la suite, à l'adoption de son enfant adoptif par son conjoint.

\section{L'adoption de l'enfant né d'une gestation pour autrui}

55 La même loi de 2002 a aussi institué la filiation fondée sur un projet parental avec assistance à la procréation. Un projet parental existe dès lors qu'une personne seule ou des conjoints décident, pour avoir un enfant, de recourir aux forces génétiques d'un tiers (art. 538 C.c.Q.). Les couples lesbiens peuvent seuls s'en prévaloir pour établir directement la 
filiation avec la femme qui n'a pas porté l'enfant, sans que l'autre doive donner un consentement spécial en sa faveur (art. 539.1 C.c.Q.). Par contre, les couples hétérosexuels ou homosexuels qui font appel à une mère porteuse doivent recourir à l'adoption pour contourner la prohibition des contrats de gestation pour autrui (art. 541 C.c.Q.). La filiation est d'abord établie avec la mère porteuse et l'homme reconnu comme le père, qui peuvent ensuite consentir à l'adoption par le conjoint ou la conjointe de cet homme. Malgré la nullité des contrats de gestation pour autrui, la Cour d'appel a fait droit à une telle requête mettant ainsi fin à une controverse jurisprudentielle sur cette question ${ }^{60}$. Cependant, l'un des parents d'intention se trouve ainsi obligé d'adopter son propre enfant comme autrefois le parent devait adopter son enfant naturel pour tenir en échec les dispositions relatives au statut d'illégitimité. C'est pourquoi plusieurs proposent un encadrement juridique des contrats de mère porteuse qui baliserait les pratiques et permettrait de reconnaître le projet parental comme fondement de la filiation pour l'établir directement avec les deux parents d'intention ${ }^{61}$.

Le petit échantillon que nous avons étudié dans le district de Saint-François comporte un seul cas d'adoption homoparentale de l'enfant du conjoint et un seul d'adoption d'un enfant apparemment né d'une gestation pour autrui. On peut rapprocher la rareté relative de ces adoptions par des parents d'intention de la semblable rareté des adoptions d'enfants naturels par leurs propres parents en 1953. Or, celles-ci sont ensuite devenues plus importantes en 1963 et en 1973, avant de disparaître quand la réforme du droit de la famille de 1980 a conféré des droits égaux à tous les enfants.

\section{Conclusion}

Ce survol des transformations de l'adoption légale et de la famille au Québec retrace le passage, d'une logique d'exclusion de l'enfant naturel de l'ordre familial légitime, à une logique égalitaire qui accorde des droits égaux à tout enfant dont la filiation est établie. Jusqu'à la fin des années 1960, l'adoption compense un traitement nettement discriminatoire de l'enfant naturel, sans lui permettre pour autant de concurrencer l'enfant légitime. Elle lui donne donc une famille, mais le garde à la marge de sa parenté adoptive. En 1969, quand l'enfant adopté est finalement légitimé, la logique patrimoniale qui s'exerçait à son encontre est devenue inopérante. Dorénavant, il est membre à part entière de sa parenté adoptive du seul fait qu'il est légalement reconnu comme l'enfant de ses parents adoptifs. Un nouveau modèle de famille biparentale prévaut. Puis, en 1980, un renversement se produit. L'adoption entre véritablement dans un régime substitutif qui échange une filiation pour une autre, sans permettre de chevauchement entre les deux (sauf en ce qui concerne les empêchements de mariage avec la famille d'origine). Elle se trouve ainsi à exclure, à chaque fois, les parents d'origine et leur parenté. L'adoption légale est ainsi révélatrice d'une tension autrement peu perceptible entre, d'une part, un paradigme juridique tout à fait égalitaire et, d'autre part, un principe d'exclusivité de la filiation qui écarte d'un ordre familial pourtant de plus en plus électif toutes les figures parentales autres que les deux parents légaux.

L'accent mis sur la dimension affective de l'adoption et l'absence d'alternative à l'adoption plénière portent à examiner l'intérêt de l'enfant adopté seulement sous l'angle de son intégration à sa nouvelle famille, sans poser la question de son intérêt éventuel au maintien de ses liens familiaux antérieurs. Pourtant, certains enfants adoptés connaissent leurs parents d'origine et ont parfois vécu avec eux pour des périodes plus ou moins longues. C'est aussi le cas de la plupart des enfants qui sont adoptés par de proches parents ou par le nouveau conjoint de leur père ou de leur mère et qui doivent changer d'état civil tout en restant au sein de la même famille. Étant donné ces divers cas, l'introduction en droit québécois d'une forme d'adoption additive ou inclusive, qui ne romprait pas la filiation d'origine de l'enfant adopté, aurait sa raison d'être. Cette proposition a fait partie d'un projet de réforme du régime québécois de l'adoption amorcé 
en $2007^{62}$. Elle a été reprise dans un avant-projet de loi soumis en $2009^{63}$, puis dans deux projets de loi qui sont morts au feuilleton, l'un en 2012 et l'autre en $2013^{64}$. Cependant, entre 2007 et 2013, elle a été progressivement réduite à la proposition de ne maintenir qu'une inscription nominale du parent significatif, sans préserver le lien de parenté entre lui et son enfant ${ }^{65}$. L'occasion de permettre un cumul de filiations n'a pas été saisie.

Si l'on ne tient pas compte de l'adoption intrafamiliale, parce qu'on la croit trop marginale, l'évolution du droit de l'adoption et de la famille semble n'être qu'une marche continue vers l'égalité de tous les parents et de tous les enfants. Il a fallu porter attention aux particularités de l'adoption de l'enfant du conjoint (qui n'entraînait pas la perte de la légitimité antérieure de l'adopté entre 1969 et 1980) pour saisir que la réforme du droit familial de 1980 a opéré un véritable renversement dans la manière de penser les frontières de la famille et de la parenté. Ce renversement explique, sans doute en partie, le fait que la reconnaissance de parentés plurielles demeure encore difficilement envisageable en droit québécois.

\section{Notes}

1 Cette recherche réalisée sous la direction de Carmen Lavallée a bénéficié d'un soutien financier de la Chambre des notaires du Québec.

2 Le Québec compte 36 districts judiciaires. En 2012, celui de Saint-François comptait 281733 personnes (https://www.barreau.qc.ca/pdf/gouvernance-ordre/conseil-general-tableau-nbmembres-population- section.pdf).

3 Avec la permission du tribunal, nous avons étudié les dossiers des années 1953, 1963, 1973, 1983, 1993, 2003 et 2008 à 2014.

4 En 1921, la Loi de l'assistance publique a réparti par tiers la charge financière entre le gouvernement provincial, les municipalités et les institutions de charité (Micheline Dumont, « Des religieuses, des murs et des enfants ", L'Action nationale, vol. 84, no 4, 1994, p. 483-508).

5 En 1941, 61\% des Québécois vivent en milieu urbain ; en 1971, 74\%. Voir p. 35 dans Marie-Paule Malouin, L'univers des enfants en difficulté au Québec entre 1940 et 1960, Boucherville, Éditions Bellarmin, 1996.

6 Chantal Collard, « Enfants de Dieu, enfants du péché ; anthropologie des crèches québécoises de 1900 à 1960 ", Anthropologie et Sociétés, 12 (2), 1988, p. 97-123.

7 Après la conquête britannique de la Nouvelle-France, en 1760, les Canadiens-français ont conservé leur système de lois en matière civile dérivant de la Coutume de Paris, auquel se sont ajoutées des modifications par les statuts de la province et d'autres règles empruntées au droit anglais. Ce droit de sources mixtes a été codifié dans le Code civil du Bas-Canada entré en vigueur en 1866 (ci-après C.c.B.C.), juste avant la création de la confédération canadienne (1867). Marcel Guy, « Le Code civil du Québec : un peu d'histoire, beaucoup d'espoir », Revue de droit de l'Université de Sherbrooke, no 23, 1993, p. 457-492.

8 Jean-Louis Baudoin, «Examen critique de la situation juridique de l'enfant naturel », McGill Law Journal 12, 1966, p. 157-182.

9 Art. 165 et 168 du C.c.B.C.

10 Le mariage seul suffit, sans reconnaissance préalable, mais la légitimation n'a pas d'effet rétroactif. Elle n'est pas permise pour l'enfant adultérin ou incestueux.

11 Restreinte aux aliments, dans le cas de l'enfant adultérin ou incestueux.

12 Baudoin (op. cit. p. 158) parle de la famille naturelle comme d'une famille «para-juridique ».

13 Selon Malouin (op. cit. p. 95-98), 80\% à 85\% des filles-mères confient leur enfant en adoption entre les années 1920 et 1960.

14 La Loi sur les mères nécessiteuses de 1937 ne vise que les veuves ayant au moins deux enfants et d'une moralité irréprochable.

15 Marie-Aimée Cliche, «Les filles-mères devant les tribunaux de Québec 1850-1969 », Recherches sociographiques, vol. XXXII, no 1, 1991, p. 9-42.

16 Sur les idéologies de la parenté au Québec avant 1960, voir Chantal Collard, op. cit.

17 L'expression est apparue vers 1870. Voir Andrée Lévesque, La norme et les déviantes. Des femmes au Québec pendant l'entre-deux-guerres, Montréal, Éditions du Remue-Ménage, 1989. 
18 Loi concernant l'adoption, 14 Geo. V, 1924, c. 75.

19 Loi concernant l'adoption S.R. 1925, c.196. 1925. Voir Dominique Goubau et Claire O’Neill, "L'adoption, l'Église et l'État : les origines tumultueuses d'une institution légale », Les Cahiers de droit, Volume 38, numéro 4, 1997, p. 769-804.

20 Hervé Roch, L'adoption dans la province de Québec, Montréal, Wilson et Lafleur, 1951, à la p. 25.

21 H. Roch, ibid., p. 141.

22 Léon Roy, De la tenue des registres de l'état civil dans la province de Québec, Québec, 1959, p. 68.

23 Voir Carmen Lavallée, «L'adoption québécoise : une oscillation perpétuelle entre la continuité et la rupture ", dans La personne humaine, entre autonomie et vulnérabilité, sous la direction de Christelle Landheer-Cieslak et Louise Langevin, Montréal, Éditions Yvon Blais, 2015, p. 331-364. Pour Mireille Castelli, « La nouvelle loi sur l'adoption », Cahiers de droit 13, 1972, p. 567, l'adoption ne crée pas un véritable lien de filiation. Pour Albert Mayrand, "Adoption et successibilité ", Revue du Barreau 9, 1959, p. 409, elle crée une double appartenance familiale.

24 Entre 1940 et 1960, de 2,7\% à 3,6\% des naissances sont illégitimes (une proportion inférieure à celle du reste du Canada), mais leur nombre absolu augmente (Malouin, op. cit. p. 118).

25 Micheline Dumont, "Des religieuses, des murs et des enfants », L'Action nationale, vol. 84 (4), 1994, p. 483-508. Dès 1948, les deux tiers des mères confient leur enfant illégitime à l'adoption.

26 Pour Jean Pineau « La situation juridique des enfants nés hors mariage », Thémis, vol. 8 (2), 1973, p. 209-222, c'est " une véritable légitimation, en-dehors de tout mariage ». Toutefois, l'adoption n'avait pas les mêmes effets que la légitimation.

27 Baudoin mentionne que la Loi sur l'adoption a permis aux enfants adultérins d'être adoptés par leurs propres parents et de tourner ainsi les prohibitions des art. 606 et 768 du C.c.B.C. (op. cit. p. 161).

28 Paul Paquette, «La loi de l'adoption : ses motifs, sa nature, ses effets », La Revue du Notariat 65, XXVIII, 1925, à la p. 83.

29 Michael Gauvreau, The Catholic Origins of Quebec's Quiet Revolution, 1931-1970, Montreal \& Kingston, McGill-Queen's University Press, 2005.

30 Notamment, un programme d'assurance-hospitalisation, en 1961 ; la nationalisation de l'électricité en 1963 ; la création du ministère de l'Éducation en 1964 ; celle du régime des rentes en 1965 ; la Loi de l'aide sociale en 1969 ; l'assurance-maladie gratuite et universelle, en 1970.

31 D. Gauvreau et. al., op. cit. p. 31.

32 Renée B.-Dandurand, Le mariage en question, Québec, IQRC, 1988, aux pages 24-25.

33 Loi concernant le divorce. S.C. 1967-68, c. 24.

34 Loi concernant le mariage civil, L.Q. 1968, c. 82.

35 Mathieu Peter, « Métamorphoses des orphelinats québécois », Recherches sociographiques, vol. 52 (2), 2011, p. 285-315.

36 Rénald Gravel, Le vécu en adoption internationale au Québec, ministère des Affaires sociales, 1984 .

37 D. Gauvreau et al., op. cit.

38 Nicole Marcil-Gratton, "Le recours spectaculaire à la stérilisation au Québec de 1971 à 1979 ", Cahiers québécois de démographie, vol. 10 (2), 1981, p. 189-210.

39 Voir Les Cahiers de droit, 1965-66, dont des articles parlent, par exemple, du passage de la famille groupe à la famille ménage, de la famille souche à la famille foyer, de la famille traditionnelle à la famille moderne.

40 Denise Lemieux et Michelle Comeau, Le mouvement familial au Québec 1960-1990, Sainte-Foy, PUQ, 2002. Aussi, Renée B. Dandurand et Françoise-Romaine Ouellette, "Famille, État et structuration d'un champ familial », Sociologie et Sociétés XXVII (2), 1995, p. 103-119.

41 Loi modifiant la Loi de l'adoption, S.Q. 1959-69, c.10.

42 Loi de l'adoption, L.Q. 1969, c. 64.

43 Or, le mari est présumé être le père de l'enfant (art. 218 C.c.B.C.). Cet article $7 \mathrm{f}$ " présente une difficulté d'interprétation quasi insurmontable » (voir p. 325 de Monique Lauzon, « Réflexions sur l'adoption », Thémis no 5, 1970, p. 323-337).

44 Pour une analyse détaillée voir Carmen Lavallée, op. cit.

45 Édith Deleury-Bonnet, « La loi de l'adoption », Les Cahiers de droit 10 (4), 1969, p. 759-775.

46 L.Q. 1977, c. 20. 
47 Nicole Roy, «L'autorité parentale et l'obligation alimentaire des parents envers leur enfant : deux institutions proposant une conception de l'intérêt de l'enfant et de la famille », Revue du Barreau 61, 2001, p. 51-183. Voir notamment p. 70-76.

48 L.Q. 1980, c.39.

49 L.R.Q., c.C-12.

50 En 1996, l'obligation entre grands-parents et petits-enfants sera même abolie. Loi modifiant le Code civil en matière d'obligation alimentaire, L.Q.1996, c.28.

51 Sur le sujet, voir Alain Roy, Le droit de l’adoption au Québec, Montréal, Wilson \& Lafleur, 2006.

52 L'âge se réduit à 14 ans au moment de l'entrée en vigueur du Code civil de 1994.

53 Françoise-Romaine Ouellette, L'adoption. Les acteurs et les enjeux autour de l'enfant, SainteFoy : IQRC/PUL, 1996.

54 En 1993, 717 cas et en 2003, 908 ; mais seulement 218 en 2013 (adoption.gouv.qc.ca).

55 Françoise-Romaine Ouellette et Dominique Goubau, « Entre protection et captation. L'adoption québécoise en Banque mixte », Anthropologie et Sociétés, 33 (1), 2009, p. 65-81.

56 Cette caractéristique rapproche l'adoption québécoise de l'adoption autochtone qui présente aussi ce cas de figure. Voir Carmen Lavallée, " L'adoption coutumière et l'adoption québécoise ; vers l'émergence d'une interface entre les deux cultures », Revue générale du droit 4, 2001, p. 655-702.

57 Peu de recherches en parlent. Pour la France, voir Agnès Martial, S'apparenter : ethnologie des familles recomposées, Paris, Maison des Sciences de l'Homme, 2003.

58 Le tribunal peut toutefois requérir une telle évaluation s'il le juge nécessaire.

59 L.Q. 2002, c.6. Voir, par exemple, Pierre-Claude Lafond et Brigitte Lefebvre (dir.), L'union civile, nouveaux modèles de conjugalité et de parentalité au 21 e siècle, Cowansville, Yvon Blais, 2003.

60 Adoption - 1445, 2014 QCCA 1162 (C .A .).

61 Voir, notamment, p. 166-186 de Comité consultatif sur le droit de la famille, Alain Roy (prés.), Pour un droit de la famille adapté aux nouvelles réalités conjugales et familiales, ministère de la Justice du Québec, 2015. Aussi, Marie-France Bureau et Édith Guilhermont, « Maternité, gestation et liberté ; réflexion sur la prohibition de la gestation pour autrui en droit québécois », Revue de droit et santé de McGill vol. 4 (2), 2011, p. 45-76.

62 Groupe de travail sur le régime québécois de l'adoption (prés. par Carmen Lavallée), Pour une adoption à la mesure de chaque enfant, Québec, 30 mars 2007.

63 Loi modifiant le Code civil et d'autres dispositions législatives en matière d'adoption, d'autorité parentale, avant- projet de loi, 1e sess., 39e lég. Qc.

64 Loi modifiant le Code civil et d'autres dispositions législatives en matière d'adoption, d'autorité parentale et de divulgation de renseignements (PL 47), $1^{\text {re }}$ sess. 40 légis., Qc. ; Loi modifiant le Code civil et d'autres dispositions législatives en matière d'adoption, d'autorité parentale (PL 81), $2 \mathrm{e}$ sess.,

39e lég. Qc.

65 Françoise-Romaine Ouellette et Carmen Lavallée, « La réforme proposée du régime québécois de l'adoption et le rejet des parentés plurielles », McGill L.J. 60 (2), p. 295-331.

\section{Pour citer cet article}

Référence papier

Françoise-Romaine Ouellette et Carmen Lavallée, « L'adoption légale comme révélateur de l'évolution de la famille au Québec », Droit et cultures, 73 | 2017, 49-68.

Référence électronique

Françoise-Romaine Ouellette et Carmen Lavallée, « L'adoption légale comme révélateur de l'évolution de la famille au Québec », Droit et cultures [En ligne], 73 | 2017-1, mis en ligne le 23 mars 2017, consulté le 13 novembre 2019. URL : http://journals.openedition.org/droitcultures/4062

\section{Auteurs}

Françoise-Romaine Ouellette

Françoise-Romaine Ouellette est anthropologue, professeure titulaire à l'Institut national de la recherche scientifique, Centre Urbanisation Culture Société. Ses travaux portent sur les 
conceptions, normes et valeurs relatives à la famille et à la filiation. Elle s'est intéressée principalement à l'adoption. Elle a publié avec Julie Saint-Pierre en 2011, « Parenté, citoyenneté et état civil des adoptés ", Enfance, famille, générations n 14, p. 51-76 ; en 2009, "The social temporalities of adoption and limits of plenary adoption», dans D. Marre et L. Briggs, International Adoption: Global Inequalities and the Circulation of Children, New York, New York University Press, p. 69-86 ; en 2008, "Les noms et papiers d'identité des enfants adoptés à l'étranger », dans Agnès Fine (dir.), États civils en question. Papiers, identités, sentiment de soi, Paris, Éditions du CTHS, Le regard de l'ethnologue n¹9, p. 147-174. Francoise-Romaine.Ouellette@UCS.INRS.Ca

\section{Carmen Lavallée}

Carmen Lavallée est avocate et professeure à la Faculté de droit de l'Université de Sherbrooke. Ses travaux portent principalement sur la protection de l'enfant privé du soutien de sa famille en droit interne et en droit international. Elle s'intéresse également aux différentes règles normatives régissant la famille et la filiation. Elle a publié notamment : La protection internationale des droits de l'enfant : entre idéalisme et pragmatisme, Bruxelles, Brylant, coll. « Droit et mondialisation », 2015, L'enfant, ses familles et les institutions de l'adoption, Regards sur le droit français et le droit québécois, Montréal, Wilson et Lafleur, 2005 ; « Homoparenté, parentalité et filiation en droit québécois : une égalité à géométrie variable », (2012) 1 RIDC 13-34, "Pour une adoption dans rupture du lien de filiation d'origine dans les juridictions de civil law et de common law » (2008) 146 Informations Sociales 132-141. Carmen.Lavallee@USherbrooke.ca

Articles du même auteur

La situation juridique des conjoints de fait québécois [Texte intégral]

Legal Situation of de facto Spouses in Quebec Civil Law

Paru dans Droit et cultures, 73 | 2017-1

\section{Droits d'auteur}

\section{(c) (i) $(9)$}

Droits et Culture est mis à disposition selon les termes de la licence Creative Commons Attribution Pas d'Utilisation Commerciale - Pas de Modification 4.0 International. 